\title{
RECENZJA: DOMENICA ELISA CICALA: UMORISMO ANTE LITTERAM LA CONCEZIONE UMORISTICA PIRANDELLIANA IN OPERE NARRATIVE ANTERIORI AL 1908, ROMANISTISCHER VERLAG, BONN 2009, ss. 480.
}

Domenica Elisa Cicala, docente di letteratura italiana presso l'Università di Klagenfurt in Austria, nel suo saggio intitolato Umorismo ante litteram. La concezione umoristica pirandelliana in opere narrative anteriori al 1908, compie una ricognizione esaustiva e attenta del fenomeno dell'umorismo definito da Luigi Pirandello. Le sue analisi si concentrano sulle opere apparse prima della pubblicazione dell'Umorismo, dunque prima del 1908, ciò pertanto viene suggerito dallo stesso titolo.

Il libro, a prima vista, si presenta ricco di diversi contenuti; si tratta di un bel volume che contiene 480 pagine. Esso è suddiviso in tre lunghe parti, intitolate rispettivamente: La concezione umoristica pirandelliana, Umorismo ante litteram nei primi romanzi, L'umorismo in novelle anteriori al 1908. Alla fine del lavoro troviamo una breve conclusione, l'appendice e le note bibliografiche. La bibliografia include studi in francese, inglese, italiano e tedesco. Essi sembrano molto utili per chi vuole continuare il lavoro iniziato da Cicala e approfondire le conoscenze sulla visione dell'umorismo pirandelliano.

La prima parte (pp. 19-105) costituisce la base teorica del libro e serve da pilastro per le analisi testuali condotte nella prosecuzione del saggio. Le riflessioni di Cicala ruotano non solo intorno agli autori, che hanno probabilmente influito su Pirandello (Bachtin, Bergson, Binet, Freud), ma definiscono anche la nozione dell'umorismo prendendo in considerazione vari fattori relativi alla vita 
privata del «grande agrigentino». Vi sono dunque molti rinvii alla biografia di Pirandello.

L'autore, con una notevole attenzione e precisione, analizza il più importante saggio pirandelliano, facendo riferimenti alla sua struttura ed alle informazioni significative ivi comprese e trasmesse. Attraverso l'umorismo, pare dirci giustamente la studiosa, Pirandello arriva incoscientemente ad indicare i confini della propria poetica. Come sottolinea Cicala, esso svolge un ruolo cruciale nella maggior parte della sua produzione letteraria.

Nella seconda parte (pp. 107-395) l'autore focalizza la sua attenzione sulle caratteristiche generali del corpus romanzesco pirandelliano, individuandone gli elementi strutturali (personaggi, temi, tempo, spazio ecc.) e sullo studio dei primi tre romanzi in chiave umoristica (L'esclusa, Il turno, Il fu Mattia Pascal). Le analisi testuali delle opere sopra elencate, per raggiungere gli obiettivi prefissati, sono condotte secondo la prospettiva della presenza dell'umorismo. Cicala, servendosi di nozioni dicotomiche come ad esempio vita/forma, comico/tragico, bello/brutto, realtà/ finzione, essere/apparire, riesce a provare che il «sentimento del contrario» in cui consiste l'umorismo è l'elemento principale e costitutivo della trama delle opere pirandelliane prese in esame. Proprio sulla concezione umoristica, afferma la studiosa, si incentra il perno dei primi romanzi di Pirandello. Essa si percepisce a vari livelli della loro struttura ed è applicata alle tecniche narrative che si rivelano imbevute di umorismo.

La seconda parte, tranne una dettagliata analisi testuale, verte su riassunti minuziosi e completi dei lavori ivi studiati. Tale tipo di struttura, occorre sottolinearlo, appare di rado negli studi critici, ma è di gran rilievo poiché dà al lettore la possibilità di confrontare il contenuto dell'opera con l'interpretazione dell'autore senza la necessità di riferirsi al testo originale.

Con l'ultima parte ( pp. 397-445) ci si inoltra nel mondo delle novelle pirandelliane scritte prima del 1908. Vi sono annoverati i loro tratti generali e poi l'autore passa a testi concreti, il che è in sintonia con l'ordine strutturale della seconda parte. Ambedue le parti sono dunque strutturate secondo lo stesso modello per cui il lavoro diventa più leggibile e coerente.

La produzione novellistica di Pirandello, come nota la studiosa, s'imbeve di connotati umoristici. Essi di nuovo sono inerenti alla quotidianità dell'individuo la quale si dimostra strana, piena di paradossi, falsa e vuota. Da quanto si evince dalle novelle analizzate la vita risulta assurda e priva di senso. Cicala fa un resoconto agile ed intelligente delle situazioni in cui si può percepire la presenza dell'umorismo. Le sue analisi sono, però, un po' più superficiali di quelle condotte nella seconda parte del saggio, ma sembrano molto interessanti. 
Un altro aspetto meritevole del lavoro di Cicala è nell' appendice, dove si trova una lettera autobiografica di Pirandello in cui lo scrittore parla delle sue opere determinandone il carattere. Esso è, come rileva lo stesso «figlio del Caos», di matrice umoristica. Pare che questa lettera venga citata per confermare ancora una volta le conclusioni cui si è arrivati. La lettera è seguita da un elenco delle novelle pirandelliane anteriori al 1908 presentate in ordine cronologico secondo il titolo e la data.

In conclusione, si tratta di un lavoro scrupoloso e profondo nella problematica della materia trattata. La lettura di esso è fortemente consigliata agli studenti e studiosi che sono interessati ad esplorare il mondo della prosa di Pirandello. Cicala ne tratteggia un quadro completo in cui spicca la presenza dell'umorismo. Il suo saggio, considerando la scarsità di lavori dedicati allo studio della concezione umoristica pirandelliana, riempie un vuoto nelle ricerche su questo tema. 